\title{
Linking bulk modulus to an unilateral damage yield criterion: a thermodynamic modeling approach

\section{P. Saramito ${ }^{1}$, V. Dansereau ${ }^{2,3}$ and J. Weiss ${ }^{2}$}

\begin{abstract}
This work presents a new damage criterion suitable for elastic, elastic-plastic/viscous or elastic-viscous-plastic materials involving rupture effects. Its derivation, made here within a thermodynamic framework, follows previous scalar-valued damage mechanics approaches. Such approaches are appropriate to many geophysical problems involving quasi-brittle materials for which there is no clear physical justification for the level of complexity of a tensorial damage variable. Distinction between the mechanical response to compressive and tensile stresses is therefore not introduced by the damage itself but via a special definition of the Helmholtz free energy. This scheme differs from previous ones in that it combines with an evolution of Poisson's ratio with the level of damage, which allows expressing the damage criterion in the principal stresses space. Moreover, there is no need to compute the stress eigensystem, which makes it simpler to implement than the Mohr-Coulomb damage criterion.

Here we derive this damage criterion and compare it to observations of the variations of the bulk modulus in damaged geomaterials. We also compare it to in-situ stress measurements and find a good agreement in terms of the shape of the criterion in the stress space. We tentatively interpret the results in the context of previous studies of rock and ice mechanics.
\end{abstract}

\section{Keywords}

Damage mechanics, thermodynamics 


\section{Introduction}

Modern continuum damage mechanics is funded on the seminal work of Kachanov (1958), who, in order to predict the creep rupture of metals, introduced the concept of a macroscopic, continuous damage scalar field, $d$, that describes the evolution of cracks at the microscopic scale and their impact on macroscopic elasticity. This continuous approach was rapidly applied to many materials such as concrete, polymers and rocks, then combined with various local failure criteria to model progressive damage within heterogeneous materials. In geosciences, in particular, the combination of the damage mechanics of Kachanov (1958) and of the Mohr-Coulomb criterion (Coulomb 1773; Mohr 1900; Jaeger et al. 2007) is widely used to predict the localization of damage and subsequent failure of natural materials at the laboratory or geophysical scale (see e.g. Amitrano et al. 1999; Amitrano 2003; Girard et al. 2011; Riva et al. 2018).

Continuum damage mechanics, initially funded on phenomenology, evolved in the last decades towards a more complete theory thanks to the thermodynamics of irreversible processes, which introduced internal variables together with the concept of the dissipation potential (see Lemaitre 1985; Lemaitre and Chaboche 1990; Murakami 2012). In thermodynamics, the strain energy release rate, $Y$, used for the damage criterion is elegantly introduced by duality from the damage variable $d$. This concept, set forth by Erdogan and Sih (1963), is a natural and obvious generalization of Griffith's (1921) original energy release rate that explains the extension of cracks in a material. It constitutes an important change of paradigm in the context of the previous Mohr-Coulomb damage criterion, which is rather formulated in terms of stresses and is derived from a theory of the friction along already existing interfaces that does not clearly explain the formation of new cracks.

Initial thermodynamically-based damage theories have postulated that damage evolves in the same way under tensile and compressive stresses. This is a strong shortcoming, as micro-crack nucleation and propagation mechanisms strongly differs between tensile and compressive stress states, for both porous (e.g. Sammis and Ashby 1986) and non-porous (Ashby and Sammis 1990) materials.

\footnotetext{
${ }^{1}$ LJK, CNRS and Grenoble univ., France

${ }^{2}$ Isterre, CNRS and Grenoble univ., France

${ }^{3}$ present adress: L3SR, CNRS and Grenoble univ., France
}

\section{Corresponding author:}

P. Saramito

Email: Pierre.Saramito@imag.fr 
In addition, in materials such as concrete and rocks, however, the effect of preexisting crack closure, due to compression, on elastic stiffness, i.e. on damage, can not be disregarded (Lemaitre 1985, p. 84). Ladevèze and Lemaitre (1984) first proposed an original unilateral thermodynamically-based criterion based on a scalar damage field, which distinguished between traction and compression (see also Lemaitre 1996, p. 80; Murakami 2012, p. 101 or Besson et al. 2010, p. 163). More sophisticated anisotropic approaches have been considered. These take into account the orientation of cavities and micro-cracks through the introduction of second rank damage symmetric tensors that replace the scalar damage variables $d$ and $Y$ (see Hayakawa and Murakami 1997; Kondo et al. 2007; Murakami 2012). Such tensor-valued damage approaches however introduce new internal material parameters that can be hard to quantify and therefore make them difficult to constrain from experimental data and to implement in practice.

The aim of the present paper is to extend, within a thermodynamic framework, the simpler scalar-valued damage field approach proposed by Ladevèze and Lemaitre (1984). Here, the distinction between traction and compression is combined with an evolution of the Poisson's ratio with the level of damage. We base this evolution on the experimental observations of Heap et al. (2009, 2010), who revisited for various rocks the link between the evolution of the Young's modulus and the evolution of the Poisson's ratio at the macroscopic, i.e., continuum, scale.

The first section presents the mathematical model in the thermodynamic framework. The evolution equation for the scalar damage variable is obtained by differentiating the dissipation potential and is shown to satisfy the second principle of thermodynamics. Then, introducing the free energy, we obtain an expression of the stress tensor which involves the Poisson's ratio. The next section explores the proposed theoretical model in view of experimental measurements of the evolution of the Poisson's ratio versus the damage. The obtained damage criterion is finally expressed in the stress space (compression/shear) and compared with the well known but not thermodynamically based Mohr-Coulomb criterion. The paper closes with a comparison of both Mohr-Coulomb and the present criteria with in-situ internal stress data within sea ice.

\section{Thermodynamic modeling}

\section{The dissipation potential}

In the thermodynamic framework of generalized standard materials, damaged materials can be represented by an Helmholtz free energy and a dissipation potential (see Halphen and NGuyen 1975 or Saramito 2016, p. 222). Here, we consider two thermodynamic variables: the strain tensor and the damage variable. Extensions to temperature-dependent processes are standard in such thermodynamic framework, but are not considered here, as the focus is on damage. 
Let $\psi:(\varepsilon, d) \in \mathbb{R}_{s}^{3 \times 3} \times \mathbb{R} \mapsto \psi(\varepsilon, d) \in \mathbb{R} \cup\{\infty\}$ be the Helmholtz free energy where $\varepsilon \in \mathbb{R}_{s}^{3 \times 3}$ denotes the symmetric tensor of small strains, $d \in \mathbb{R}$ is the damage variable, represented by a scalar field, and $\mathbb{R}_{s}^{3 \times 3}$ denotes the set of symmetric $3 \times 3$ real matrices. The practical choice of Helmholtz free energy, which introduces a distinction between traction and compression, will be discussed in the next section. At this stage, we only assume that $\psi$ is lower semi-continuous with respect to the two thermodynamic variables $(\varepsilon, d)$ and convex with respect to $\varepsilon$. The dissipation potential is denoted by $\phi:(\dot{\varepsilon}, \dot{d}) \in \mathbb{R}_{s}^{3 \times 3} \times \mathbb{R} \mapsto \psi(\varepsilon, d) \in \mathbb{R} \cup\{\infty\}$ where $(\dot{\varepsilon}, \dot{d})$ are the rates of the two thermodynamic variables. In the present paper, we are interested by damage criteria involving yield effects. One of the simplest dissipation potential involving a yield value writes as:

$$
\phi([d] ; \dot{\varepsilon}, \dot{d})=\frac{\beta_{d}}{(1+n)(1-d)}|\dot{d}|^{1+n}+Y_{c}|\dot{d}|
$$

where $Y_{c} \geqslant 0$ is the yield strain energy release rate value, that has the dimension of a stress, $n>0$ is a power index and $\beta_{d}>0$ is a damage constant that has the dimension of a viscosity. For a discussion upon $Y_{c}$, see e.g. Lemaitre (1996), p. 69, Murakami (2012), p. 98 or recently Berthier et al. (2017). In (1), the notation with square brackets $[d]$ in the left-hand-side indicates a dependence of the dissipation potential upon $d$ as parameters: it is distinct from $\dot{d}$ which is a thermodynamic rate variable of the dissipation potential.

Remark 1. Additional dissipative processes.

Note that the dissipation potential $\phi$ given by (1) does not depend on the rate of deformation tensor $\dot{\varepsilon}$. Indeed, $\phi$ vanishes when $\dot{d}=0$ for any value of the rate of deformation tensor $\dot{\varepsilon}$. This implies that here the dissipation takes its origin in the damage process only. There is therefore no dissipation due to viscous or plastic effects: the material is hyperelastic with damage effects. This choice is done for the sake of simplicity, as the focus here is on damage itself. Additional dissipative terms such as $\sigma_{y}|\dot{\varepsilon}|$ or $\eta_{0}|\dot{\varepsilon}|^{2}$, where $\eta_{0}>0$ and $\sigma_{y}>0$ are the viscosity and yield stress constants, respectively, could be easily added to $\phi$ in the righthand-side of (1) to take into account elastoplastic or viscoelastic effects. Both these terms could also be added to represent an elastoviscoplastic damageable materials (see e.g. Saramito 2016, chap. 5). Moreover, since $\phi$ is independent upon $\dot{\varepsilon}$. we simply denote $\phi([d] ; \dot{d})$. This implies that damage can occur even if no deformation occurs, for instance, if the material is purely incompressible.

Theorem 2. Second principle.

Let a material be defined by its free energy $\psi$ and its dissipation potential $\phi$ be given by (1). Then the material satisfies the second principle of thermodynamics.

Proof: Note that $\phi$ is convex with respect to the two thermodynamic rate variables $(\dot{\varepsilon}, \dot{d})$ because the absolute value function is convex. Then, from Saramito (2016), p. 223, the present material satisfies the second principle of thermodynamics. 
Note that, by its definition, which is based on a dissipation potential, the material also satisfies an extended Onsager symmetry principle (see eqn (5) in Halphen and NGuyen 1975).

The constitutive equations are obtained by derivation of the specific free energy and the potential of dissipation:

$$
\begin{aligned}
\boldsymbol{\sigma} & =\rho \frac{\partial \psi}{\partial \varepsilon}(\varepsilon, d) \\
0 & \in \rho \frac{\partial \psi}{\partial d}(\varepsilon, d)+\frac{\partial \phi}{\partial \dot{d}}([d] ; \dot{\boldsymbol{\varepsilon}}, \dot{d})
\end{aligned}
$$

where $\rho>0$ is the density.

Here, (2a) defines the Cauchy stress tensor, $\boldsymbol{\sigma}$, and is the usual definition of an hyperelastic material. Classically, the strain energy release rate $Y$ is defined from the Helmholtz free energy by (see e.g. Lemaitre 1996, p. 41 or Murakami 2012, p. 96):

$$
Y \quad \in-\rho \frac{\partial \psi}{\partial d}(\varepsilon, d)
$$

The damage evolution relation (2b) can also be expressed in an explicit manner by introducing the convex conjugate $\phi^{*}$ of the dissipation potential $\phi$, defined for all $Y \in \mathbb{R}$ by the Fenchel transformation:

$$
\phi^{*}([d] ; Y)=\inf _{\dot{\delta} \in \mathbb{R}}(\dot{\delta} Y-\phi([d] ; \dot{\delta}))
$$

From (4), we have classically (Rockafellar 1974, p. 35):

$$
Y \in \frac{\partial \phi}{\partial \dot{d}}([d] ; \dot{d}) \Longleftrightarrow \dot{d} \in \frac{\partial \phi^{*}}{\partial Y}([d] ; Y)
$$

With the choice (1) for the dissipation potential $\phi$, we obtain an explicit expression of its subdifferential:

$$
\frac{\partial \phi}{\partial \dot{d}}([d] ; \dot{d})= \begin{cases}{\left[-Y_{c}, Y_{c}\right]} & \text { when } \dot{d}=0 \\ \left\{Y=\frac{\beta_{d}}{1-d}|\dot{d}|^{-1+n} \dot{d}+Y_{c} \frac{\dot{d}}{|\dot{d}|}\right\} & \text { otherwise }\end{cases}
$$

Note also that the subdifferential is multi-valued when $\dot{d}=0$. Conversely, when $\dot{d} \neq 0$, it contains only one value and $\phi$ is differentiable. From (5) and (6), we obtain after few computations that the subdifferential $\partial \phi^{*} / \partial \dot{d}([d] ; \dot{d})$ of the convex conjugate $\phi^{*}$ contains exactly one element. Then, by definition, $\phi^{*}$ is differentiable and this element is precisely its gradient, denoted by $\nabla \phi^{*}([d] ; Y)$ 
which is given by

$$
\nabla \phi^{*}([d] ; Y)= \begin{cases}0 & \text { when } Y \leqslant Y_{c} \\ \left(\frac{1-d}{\beta_{d}}\left(|Y|-Y_{c}\right)\right)^{\frac{1}{n}} \frac{Y}{|Y|} & \text { otherwise }\end{cases}
$$

Note again that, by integration, we obtain an explicit expression of the convex conjugate $\phi^{*}$ :

$$
\phi^{*}([d] ; Y)=\frac{1}{1+\frac{1}{n}}\left(\frac{1-d}{\beta_{d}}\right)^{\frac{1}{n}} \max \left(0,|Y|-Y_{c}\right)^{1+\frac{1}{n}}
$$

Combining (2b), (3) and (5), leads to an explicit expression of the damage evolution equation:

$$
\dot{d}=\nabla \phi^{*}([d] ; Y)
$$

Note that the damage criterion appears now explicitly in the previous damage evolution equation, via the expression (7a) of its right-hand-side. Indeed, when $|Y| \leqslant Y_{c}$, from (7a)-(7b), we get $\dot{d}=0$ i.e. damage ceases to evolve.

Proposition 3. Explicit expression of the dissipation.

The dissipation, defined by $\mathscr{D}=Y \dot{d}$, is always positive. It expresses as

$$
\begin{aligned}
\mathscr{D} & =\frac{\beta_{d}}{1-d}|\dot{d}|^{1+n}+Y_{c}|\dot{d}| \\
& =\left(\frac{1-d}{\beta_{d}} \max \left(0,|Y|-Y_{c}\right)\right)^{\frac{1}{n}}|Y| \geqslant 0
\end{aligned}
$$

Proof: From the Clausius-Duhem principle (see e.g. Saramito 2016, p. 221), and because the process is here considered isotherm, the dissipation reads

$$
\begin{aligned}
\mathscr{D} & =\boldsymbol{\sigma}: \dot{\boldsymbol{\varepsilon}}-\rho \dot{\psi}(\varepsilon, d) \\
& =\boldsymbol{\sigma}: \dot{\boldsymbol{\varepsilon}}-\rho \frac{\partial \psi}{\partial \varepsilon}(\varepsilon, d): \dot{\varepsilon}-\rho \frac{\partial \psi}{\partial d}(\varepsilon, d) \dot{d} \quad \text { by expansion of } \dot{\psi} \\
& =-\rho \frac{\partial \psi}{\partial d}(\varepsilon, d) \dot{d} \text { from }(2 \mathrm{a}) \\
& =Y \dot{d} \text { from }(3)
\end{aligned}
$$

The second expression of the dissipation in (8) is a direct consequence from (6) that gives $Y$ in terms of $\dot{d}$. The last expression is obtained from (7a)-(7b), which give $\dot{d}$ in terms of $Y$. 
Remark 4. Additional dissipative processes.

Following 1, additional dissipative terms could be inserted in the expression of the dissipation potential $\phi$, to account, for instance, for plastic $\sigma_{y}|\dot{\varepsilon}|$ or viscous $\eta_{0}|\dot{\varepsilon}|^{2}$ effects. These terms would then appears in the previous expression (8) of the dissipation.

The present damage yield criterion $Y>Y_{c}$, involved in the damage evolution equation (7a)-(7b), is easy to handle from a thermodynamics point of view, as it directly satisfies the second principle. On an experimental point of view, other damage criteria based on stress can however be practical as they are directly comparable to experimental stress measurements in the principal Cauchy stress space. Weiss et al. (2007) for instance interpreted such measurements within the sea ice cover in terms of the a Mohr-Coulomb damage criterion (see Fig. 2.a). In the following, we therefore express the derived damage yield criterion, $Y>Y_{c}$, in terms of the principal Cauchy stress rather than the energy release rate, and aim to verify if it agrees with experimental data. The impatient reader could observe directly Fig. 7 below. The main tool for obtaining an agreement with experimental data is a customization of the Helmholtz free energy, $\psi$, and more precisely, of the Poisson's ratio, as a damage-dependent function.

\section{The free energy}

The Helmholtz free energy is split in two parts:

$$
\psi(\varepsilon, d)=\psi_{e}(\varepsilon, d)+\mathscr{I}_{[0,1]}(d)
$$

Here $\psi_{e}:(\varepsilon, d) \in \mathbb{R}_{s}^{3 \times 3} \times \mathbb{R} \rightarrow \psi(\varepsilon, d) \in \mathbb{R} \cup\{\infty\}$ represents the contribution to elasticity of the free energy, and is called here the elastic energy, while $\mathscr{I}_{[0,1]}$ denotes the indicator function of the set $[0,1]$, defined by:

$$
\mathscr{I}_{[0,1]}(d)= \begin{cases}0 & \text { when } d \in[0,1] \\ \infty & \text { otherwise }\end{cases}
$$

The term $\mathscr{I}_{[0,1]}(d)$ in the expression (9) of the Helmholtz free energy acts as a barrier for maintaining $d$ in $[0,1]$. Recall that the indicator function is convex but not differentiable. For an isotropic elastic material, $\psi_{e}$ expresses classically as:

$$
\psi_{e}(\varepsilon, d)=\frac{E(d)}{2 \rho\left(1+\nu_{0}\right)}\left(\varepsilon: \varepsilon+\frac{\nu_{0}}{1-2 \nu_{0}}(\operatorname{tr} \varepsilon)^{2}\right)
$$

where $E: \mathbb{R} \rightarrow \mathbb{R}$ is the Young modulus, that is a positive and strictly decreasing function of the damage $d$, and $\nu_{0}$ is the constant Poisson's ratio. Also, $\operatorname{tr}(\boldsymbol{\xi})$ denotes the trace of any matrix $\boldsymbol{\xi} \in \mathbb{R}_{s}^{3 \times 3}$ and $\boldsymbol{\xi}: \boldsymbol{\delta}$ denotes the double contracted product of any matrices $\boldsymbol{\xi}, \boldsymbol{\delta} \in \mathbb{R}_{s}^{3 \times 3}$. Let us introduce the convex conjugate $\psi_{e}^{*}$ of the elastic energy $\psi_{e}$ with respect to the strain tensor variable, defined for all 
$\boldsymbol{\sigma} \in \mathbb{R}^{3 \times 3}$ and $d \in \mathbb{R}$ by a Fenchel transformation:

$$
\psi_{e}^{*}(\boldsymbol{\sigma}, d)=\inf _{\boldsymbol{\xi} \in \mathbb{R}_{s}^{3 \times 3}}\left(\frac{\boldsymbol{\xi}: \boldsymbol{\sigma}}{\rho}-\psi_{e}(\boldsymbol{\xi}, d)\right)
$$

This convex conjugate $\psi_{e}^{*}$ is also referred to as the Gibbs elastic energy. By duality (Rockafellar 1974, p. 35), we have:

$$
\boldsymbol{\sigma} \in \rho \frac{\partial \psi_{e}}{\partial \varepsilon}(\varepsilon, d) \Longleftrightarrow \varepsilon \in \rho \frac{\partial \psi_{e}^{*}}{\partial \boldsymbol{\sigma}}(\boldsymbol{\sigma}, d)
$$

With the isotropic choice (10) for $\psi_{e}$, the convex conjugate is:

$$
\begin{aligned}
\psi_{e}^{*}(\boldsymbol{\sigma}, d) & =\frac{1}{2 \rho E(d)}\left(\left(1+\nu_{0}\right) \boldsymbol{\sigma}: \boldsymbol{\sigma}-\nu_{0}(\operatorname{tr} \boldsymbol{\sigma})^{2}\right) \\
& =\frac{1}{2 \rho E(d)}\left(\left(1+\nu_{0}\right)(\operatorname{dev} \boldsymbol{\sigma}):(\operatorname{dev} \boldsymbol{\sigma})+\frac{1-2 \nu_{0}}{3}(\operatorname{tr} \boldsymbol{\sigma})^{2}\right)
\end{aligned}
$$

where $\operatorname{dev} \boldsymbol{\xi}=\boldsymbol{\xi}-(1 / 3)(\operatorname{tr} \boldsymbol{\xi}) \boldsymbol{I}$ denotes the deviator of any matrix $\boldsymbol{\xi} \in \mathbb{R}_{s}^{3 \times 3}$ and $\boldsymbol{I}$ is the identity matrix. Since the deviator $\operatorname{dev} \boldsymbol{\xi}$ and the spherical part $(1 / 3)(\operatorname{tr} \boldsymbol{\xi}) \boldsymbol{I}$ are orthogonal in $\mathbb{R}_{s}^{3 \times 3}$, the quadratic function $\psi_{e}^{*}$ is convex if and only if $\nu_{0} \in[-1,1 / 2]$ and $E(d) \geqslant 0$ for any $d \in[0,1]$. In this case, by duality, $\psi_{e}$ is also convex with respect to the strain tensor variable, and so is the Helmholtz free energy $\psi$ given by (9).

Recall that traction corresponds to $\operatorname{tr} \varepsilon>0$ while compression is characterized by $\operatorname{tr} \varepsilon<0$. Conversely, $\operatorname{tr} \boldsymbol{\sigma}>0$ represents a tensile stress while $\operatorname{tr} \boldsymbol{\sigma}<0$ is a compressive stress. In this isotropic material, damage evolves in the same way under tensile or compressive stresses: the value of the previous expression for $\psi_{e}^{*}$ is indeed independent upon the sign of $\operatorname{tr}(\boldsymbol{\sigma})$. However, as pointed out by several authors (see e.g. Lemaitre 1996; Murakami 2012), in materials such as concrete and rocks, the effect of the closure of microcracks within the material on its macroscopic mechanical behavior can not be disregarded. Besides, the evolution of the damage in most materials, metals and composites in particular, differs between tensile and and compressive stresses. In 1984, at the UCTAM conference, Ladevèze and Lemaitre (1984) therefore proposed to split the energy in two parts, depending upon the sign of the stress components (see also Lemaitre 1996, p. 80; Murakami 2012, p. 101 or Besson et al. 2010, p. 163).

This splitting approach is modified here and adapted to the sign of the trace of the Cauchy stress tensor. The previous expression (12) of the convex conjugate $\psi_{e}^{*}$ 
is replaced by:

$$
\begin{aligned}
\psi_{e}^{*}(\boldsymbol{\sigma}, d)= & \frac{1}{2 \rho E(d)}\left(\left(1+\nu_{0}\right)(\mathbf{d e v} \boldsymbol{\sigma}):(\operatorname{dev} \boldsymbol{\sigma})\right. \\
& \left.+\frac{1-2 \nu_{+}(d)}{3} \max (0, \operatorname{tr} \boldsymbol{\sigma})^{2}+\frac{1-2 \nu_{-}(d)}{3} \max (0,-\operatorname{tr} \boldsymbol{\sigma})^{2}\right) \\
= & \frac{1+\nu_{0}}{2 \rho E(d)}(\operatorname{dev} \boldsymbol{\sigma}):(\operatorname{dev} \boldsymbol{\sigma})+\frac{1}{18 \rho K(d, \operatorname{tr} \boldsymbol{\sigma})}(\operatorname{tr} \boldsymbol{\sigma})^{2}
\end{aligned}
$$

where $\nu_{ \pm}: d \in[0,1] \mapsto \nu_{ \pm}(d) \in[-1,1 / 2]$ are two continuous expressions for the Poisson's ratio as a function of the damage, which are associated to tensile and compressive stresses according to the sign $( \pm)$. We assume that $\nu_{+}(0)=\nu_{-}(0)$ and denote by $\nu_{0}=\nu_{ \pm}(0)$. For convenience, in the second expression (13b) we have introduced the bulk modulus $K$, defined for all $d \in[0,1]$ and $q \in \mathbb{R}$ by:

$$
\begin{aligned}
K(d, q) & =\frac{E(d)}{3(1-2 \nu(d, q))}= \begin{cases}K_{+}(d) & \text { when } q>0 \\
K_{-}(d) & \text { when } q<0 \\
\min \left(K_{+}(d), K_{-}(d)\right) & \text { when } q=0\end{cases} \\
\text { with } \nu(d, q) & = \begin{cases}\nu_{+}(d) & \text { when } q>0 \\
\nu_{-}(d) & \text { when } q<0 \\
\min \left(\nu_{+}(d), \nu_{-}(d)\right) & \text { when } q=0\end{cases} \\
\text { and } K_{ \pm}(d) & =\frac{E(d)}{3\left(1-2 \nu_{ \pm}(d)\right)}
\end{aligned}
$$

Here, $q$ denotes the formal parameter in the previous $K$ and $\nu$ functions that stands for $\operatorname{tr} \boldsymbol{\sigma}$ in (13b). Note that both $\nu$ and $K$, as given by (14b) and (14a), are not differentiable with respect to $d$ when $q=0$. Nevertheless, these functions admit a generalized Clarke (1990) derivative. For all $(d, q) \in[0,1] \times \mathbb{R}$, the generalized Clarke derivative of $\nu$ versus $d$ is defined as the convex hull of all directional derivatives, i.e.:

$$
\frac{\partial \nu}{\partial d}(d, q)=\left\{\begin{array}{lr}
\left\{\nu_{+}^{\prime}(d)\right\} & \text { when } q>0 \\
\left\{\nu_{-}^{\prime}(d)\right\} & \text { when } q<0 \\
{\left[\min \left(\nu_{-}^{\prime}(d), \nu_{+}^{\prime}(d)\right), \max \left(\nu_{-}^{\prime}(d), \nu_{+}^{\prime}(d)\right)\right]} & \text { when } q=0
\end{array}\right.
$$

When $q \neq 0$, the set $\frac{\partial \nu}{\partial d}(d, q)$ contains only one value, which is the usual derivative. Otherwise, when $q=0$, this derivative is multi-valued and represented by an interval bounded by the left and right derivatives $\nu_{ \pm}^{\prime}(d)$ at $q=0$.

When the Poisson's ratio is constant, i.e. $\nu_{ \pm}(d)=\nu_{0}$ for all $d \in[0,1]$, then this modified version of $\psi_{e}^{*}$ as given by (13a), coincides with (12). Note that $\psi_{e}^{*}$, as given by (13a), coincides with (12).

The present approach shares some conceptual similarities with that proposed by Ladevèze and Lemaitre (1984). In practice however, it is different in two main aspects: 
- Ladevèze and Lemaitre (1984) based their splitting on the sign of each principal stress, which requires to compute all eigenvalues and eigenvectors of the stress tensor in each point of the domain of computation. Here, there is no need to compute the stress eigensystem. This makes the present approach simpler to implement.

- The expression $\psi_{e}^{*}$ proposed by Ladevèze and Lemaitre (1984) is incompatible with experimental observations of the dependence of the Poisson's ratio in compression versus the damage $d$. Here, the present expression (13a) can be based on experimental measurements of $\nu_{ \pm}(d)$, as shown in the next section.

From (11) and by derivation of (13b), the strain tensor $\varepsilon$ expresses in terms of the Cauchy stress tensor $\boldsymbol{\sigma}$ as:

$$
\begin{aligned}
\varepsilon & =\frac{1+\nu_{0}}{E(d)} \operatorname{dev} \boldsymbol{\sigma}+\frac{\operatorname{tr} \boldsymbol{\sigma}}{9 K(d, \operatorname{tr} \boldsymbol{\sigma})} \boldsymbol{I} \\
\Longleftrightarrow \boldsymbol{\sigma} & =\frac{E(d)}{1+\nu_{0}} \operatorname{dev} \boldsymbol{\varepsilon}+K(d, \operatorname{tr} \boldsymbol{\sigma})(\operatorname{tr} \boldsymbol{\varepsilon}) \boldsymbol{I}
\end{aligned}
$$

Tacking the trace of the previous expression leads to

$$
\operatorname{tr} \boldsymbol{\sigma}=3 K(d, \operatorname{tr} \boldsymbol{\sigma}) \operatorname{tr} \boldsymbol{\varepsilon}
$$

and then $\operatorname{tr}(\boldsymbol{\sigma})$ and $\operatorname{tr}(\varepsilon)$ share the same sign. Thus, from the definition (14b) of the bulk modulus $K$ function, we have $K(d, \operatorname{tr} \boldsymbol{\sigma})=K(d, \operatorname{tr} \varepsilon)$ and we obtain the following expression of the Cauchy stress tensor $\sigma$ in terms of the strain tensor $\varepsilon$ only:

$$
\boldsymbol{\sigma}=\frac{E(d)}{1+\nu_{0}} \operatorname{dev} \varepsilon+K(d, \operatorname{tr} \varepsilon)(\operatorname{tr} \varepsilon) \boldsymbol{I}
$$

Then, from (11) and the previous expression for $\boldsymbol{\sigma}$, we obtain by integration the following explicit expression for $\psi_{e}$ :

$$
\psi_{e}(\varepsilon, d)=\frac{E(d)}{2 \rho\left(1+\nu_{0}\right)}(\operatorname{dev} \varepsilon):(\operatorname{dev} \varepsilon)+\frac{K(d, \operatorname{tr} \varepsilon)}{2 \rho}(\operatorname{tr} \varepsilon)^{2}
$$

Theorem 5. The Helmholtz free energy.

Assume that both the Young $E$ and the bulk moduli $K_{ \pm}$are convex with respect to the damage variable $d$. Then, the Helmholtz free energy $\psi$ is convex with respect to its two state variables $\varepsilon$ and $d$ separately.

Proof: Recall that the Helmholtz free energy $\psi$ is given by (9). Since the indicator $\mathscr{I}_{[0,1]}$ is convex, the proof reduces to the study of the elastic energy $\psi_{e}$, 
given by (17), and that expands as:

$$
\begin{aligned}
\rho \psi_{e}(\varepsilon, d)=\frac{E(d)}{2 \rho\left(1+\nu_{0}\right)}(\mathbf{d e v} \varepsilon):(\mathbf{d e v} \varepsilon) & +\frac{K_{+}(d)}{2 \rho} \max (0, \operatorname{tr} \varepsilon)^{2} \\
& +\frac{K_{-}(d)}{2 \rho} \max (0,-\operatorname{tr} \varepsilon)^{2}
\end{aligned}
$$

As a linear combination of convex functions with positive coefficients, the elastic energy is clearly convex with respect to $\varepsilon$. Let us now turn to its convexity with respect to $d$. The elastic energy involves three independent terms that should be all convex with respect to $d$. The first term involves $E(d)$ which is convex by assumption. The second and third terms are convex if and only if the bulk moduli $K_{ \pm}$are convex.

Remark 6. The Helmholtz free energy.

From theorem 5, the Helmholtz free energy $\psi$ is not necessarily convex with respect to its full argument $(\varepsilon, d)$, but only to $\varepsilon$ and $d$ separately. Indeed, for damaged materials, there is no warranty for the symmetric matrix of second order derivatives of $\psi$ to be positive. In consequence, stationary states of damaged materials are not necessary stable.

\section{Linking bulk modulus and damage criterion}

Theorem 7. Shape of the damage criterion in the stress space.

The shape of the damage criterion in the stress space is an ellipse (resp. an hyperbola) if and only the derivative of the bulk modulus $K$ is decreasing (resp. increasing) versus the damage $d$. Moreover, when $K$ is strictly convex, there are exactly three possibilities, represented on Fig. 1.

case 1

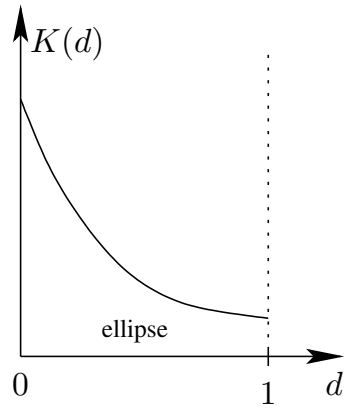

case 2

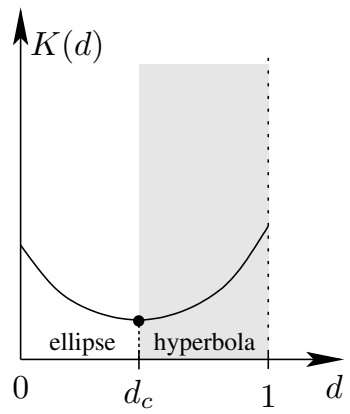

case 3

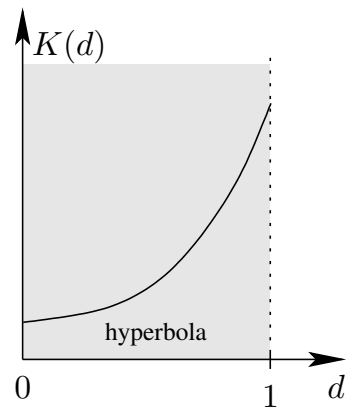

Figure 1. Variation of the bulk modulus $K$ : three possible cases when it is strictly convex. 
Proof: From (3) and (17) the strain energy release rate expresses as:

$$
Y=-\frac{E^{\prime}(d)}{2\left(1+\nu_{0}\right)}|\operatorname{dev} \varepsilon|^{2}-\frac{1}{2} \frac{\partial K}{\partial d}(d, \operatorname{tr} \varepsilon)(\operatorname{tr} \varepsilon)^{2}
$$

We also have introduced the matrix norm $|\boldsymbol{\tau}|^{2}=\boldsymbol{\tau}: \boldsymbol{\tau}$ for all $\boldsymbol{\tau} \in \mathbb{R}^{3 \times 3}$. Note that while $\partial K / \partial d$ is multi-valued when $\operatorname{tr} \varepsilon=0$, the second term in the previous expression vanishes and then $Y$ is uni-valued. In order to express the damage criterion $Y>Y_{c}$ in the stress space, it remains to replace in the previous expression of $Y$ the occurrence of $\varepsilon$ by its expression (16) in terms of $\boldsymbol{\sigma}$. Doing so, we obtain:

$$
Y=-\frac{\left(1+\nu_{0}\right) E^{\prime}(d)}{2 E^{2}(d)}|\operatorname{dev} \boldsymbol{\sigma}|^{2}-\frac{1}{18 K^{2}(d, \operatorname{tr} \boldsymbol{\sigma})} \frac{\partial K}{\partial d}(d, \operatorname{tr} \boldsymbol{\sigma})(\operatorname{tr} \boldsymbol{\sigma})^{2}
$$

The equation $Y=Y_{c}$ of the damage envelope then writes in the stress space as:

$$
\left(\frac{|\operatorname{dev}(\boldsymbol{\sigma})|}{a(d)}\right)^{2}+s(d, \operatorname{tr} \boldsymbol{\sigma})\left(\frac{\operatorname{tr}(\boldsymbol{\sigma})}{b(d, \operatorname{tr} \boldsymbol{\sigma})}\right)^{2}=1
$$

It corresponds either to an ellipse or an hyperbola in the stress space $(\operatorname{dev} \boldsymbol{\sigma}, \operatorname{tr} \boldsymbol{\sigma})$. The radii are here denoted by $a$ and $b$, while $s$ is the sign, switching from ellipse to hyperbola:

$$
\begin{aligned}
a(d) & =\frac{\sqrt{2 Y_{c}} E(d)}{\left\{-\left(1+\nu_{0}\right) E^{\prime}(d)\right\}^{\frac{1}{2}}} \\
b(d, q) & =\frac{3 \sqrt{2 Y_{c}} K(d, \operatorname{tr} \boldsymbol{\sigma})}{\left|\frac{\partial K}{\partial d}(d, \operatorname{tr} \boldsymbol{\sigma})\right|^{\frac{1}{2}}} \\
s(d, q) & =-\operatorname{sgn}\left(\frac{\partial K}{\partial d}(d, q)\right)
\end{aligned}
$$

for all $d \in[0,1]$ and $q \in \mathbb{R}$.

\section{Kachanov's damage}

Since the Young modulus $E$ is a strictly decreasing function of the damage $d$, it is a bijection between $[0,1]$ and its image by $E$, i.e. $[E(1), E(0)]$. Then, the quantities $d$ and $E(d)$ store an equivalent information when $d$ varies in $[0,1]$. Consequently, and without loss of generality, it is possible to chose, as a definition of the damage, any strictly decreasing function $E$ in $[0,1]$.

Kachanov (1958) proposed to choose the Young modulus varying linearly versus damage as:

$$
E(d)=(1-d) E_{0}
$$


where $E_{0}>0$ is the Young modulus of the undamaged material. Note that this choice of a linear dependence is not a restrictive assumption, as it can be considered as the definition of the damage variable $d$ itself: for any damaged material with a given Young modulus $\bar{E} \in\left[0, E_{0}\right]$, damage is defined as $d=1-\bar{E} / E_{0}$.

Replacing the Kachanov's expression (20) of the Young modulus $E(d)$ in the expressions (19a)-(19c) of the radii, we get:

$$
\begin{aligned}
a(d) & =\left(\frac{2 E_{0} Y_{c}}{\left(1+\nu_{0}\right) E_{0}}\right)^{\frac{1}{2}}(1-d) \\
b(d, q) & =\left(3 E_{0} Y_{c}\right)^{\frac{1}{2}} \frac{1-d}{\left.\mid \frac{1}{2}-\nu(d, q)\right)-\left.(1-d) \frac{\partial \nu}{\partial d}(d, q)\right|^{\frac{1}{2}}} \\
s(d, q) & =\operatorname{sgn}\left(\frac{1}{2}-\nu(d, q)-(1-d) \frac{\partial \nu}{\partial d}(d, q)\right)
\end{aligned}
$$

for all $d \in[0,1]$ and $q \in \mathbb{R}$.

Remark 8. Some common situations.

Assume that the Young modulus $E(d)$ is defined by (20). When Poisson's ratio is either decreasing or constant versus damage, then, from (21c), the shape of the damage envelope in the stress space is always an ellipse.

\section{Results and discussion}

\section{Bulk modulus derived from experiments in compression}

Observations of the evolution of Poisson's ratio in compression were first made by Heap et al. (2009), Fig. 7. See also Heap et al. (2010), Fig. 3, Eslami et al. (2010), Fig. 8, Grindrod et al. (2010), Fig. 9, Yang et al. (2015), Fig. 23. Abe (2016), Figs. 4 and 9 observed a behavior similar to these experiments in their discrete simulations. Several authors also reported open domains similar to hyperbolas (see e.g. Weiss et al. (2007), Fig. 13) or shifted conic domains, suggesting Mohr-Coulomb-like criteria. Our aim here is to reinterpret these experimental observations in terms of the variation of the bulk modulus versus the damage variable. Then, based on the present theory, we deduce the shape of the damage criterion in the stress space.

Fig. 2.top-left represents experimental data from Heap et al. (2010), Fig. 3.f, where a cylindrical sample of westerly granite undergoes cycles of compression. After the $i$-th cycle, $i \geqslant 1$, both the Young modulus, $\bar{E}_{i}$, and the Poisson's ratio, $\nu_{i}$, of the damaged sample are measured. Assume that the Young modulus $E(d)$ is defined 
by (20). Then, we deduce the damage variable after the $i$-th cycle as:

$$
d_{i}=1-\frac{\bar{E}_{i}}{E_{0}}
$$

We can therefore plot Poisson's ratio $\nu_{i}$ and the corresponding bulk modulus $K_{i}=E_{i} /\left(3\left(1-2 \nu_{i}\right)\right)$ as functions of the damage $d_{i}$. Figs. 2.top-right and 2.middle-right represent the obtained bulk modulus and Poisson's ratio versus the damage variable for westerly granite. Note that the bulk modulus $K_{-}$is convex and therefore, from theorem 5 , the free energy is convex with respect to the two state variables separately. Note also that the bulk modulus is a strictly increasing function of the damage: from theorem 7 , the damage criterion in the compression stress half-space is always an hyperbola. This corresponds to the case 3 on Fig. 1.

\begin{tabular}{|l||c|c|c|c|c|c|c||c|c|}
\hline & $E_{0}(G P a)$ & $E_{f}(G P a)$ & $d_{f}$ & $\nu_{0}$ & $\nu_{f}$ & $\nu_{0}^{\prime}$ & $\nu_{f}^{\prime}$ & $d_{c}$ & $\nu_{c}$ \\
\hline \hline westerly granite & 64.0 & 56.3 & 0.120 & 0.275 & 0.5 & 0.416 & 4.16 & - & - \\
\hline Etna basalt & 32.1 & 22.7 & 0.293 & 0.185 & 0.5 & 0 & 3.76 & 0.176 & 0.220 \\
\hline Darley sandstone & 20.9 & 17.4 & 0.167 & 0.076 & 0.5 & 0 & 8.96 & 0.0519 & 0.09 \\
\hline Icelandic basalt & 66.1 & 52.9 & 0.200 & 0.097 & 0.097 & 0 & 0 & - & - \\
\hline
\end{tabular}

Table 1. Model parameters used for the considered materials.

The experimental measurements of Poisson's ratio as a function of damage are approximated by a third order polynomial denoted as $\nu_{-}(d)$ and represented with a line Fig. 2.middle-right. The third order polynomial approximation is uniquely determined by its values and derivative at $d \in\left\{0, d_{f}\right\}$, provided in Table 1 . Next, relation $(14 \mathrm{c})$ is applied in order to obtain, from $\nu_{-}(d)$, an approximation $K_{-}(d)$ of the bulk modulus. Finally, we compute $\nu_{-}$and its derivative for any $d \in\left[0, d_{f}\right]$ and then evaluate the radii $a_{-}(d)$ and $b_{-}(d)$ from (21a)-(21b): Fig. 2.middle-left presents the envelope of the damage criterion for various damage values while Figs. 2.bottom shows the radii. In traction, experiment shows that the damage envelopes present an ellipsoidal shape (see e.g. Lemaitre and Chaboche 1990, p. 183). Consequently, we consider here, for simplicity, that the Poisson's ratio is constant in traction, i.e. $\nu_{+}(d)=\nu_{0}$. Indeed, from remark 8 , we recover in this case an ellipsoidal shape of the damage criterion. Observe the ellipse shape in the traction half-plane, which shrinks when damage increases. Conversely, in the compression half-plane, the shape is an hyperbola: the opening angle of this hyperbola increases with damage. Note that the damage envelop is open and unbounded in the compression half-plane: the situation is similar to the case of the Mohr-Coulomb criterion and qualitatively in agreement with experimental observations, as pointed out in the introduction. This asymmetry of the damage envelop between dilatation and compression half-planes is the major feature of the present unilateral damage model.

Let us turn to an other material, an Etna basalt, presented on Fig. 3. Poisson's ratio, shown on Fig. 3.middle-right, is approximated here by a spline function. This 
spline function is composed of two third order polynomials with a continuously derivable junction at $d=d_{c}$ :

$$
\nu_{-}(d)= \begin{cases}a_{1} d^{3}+b_{1} d^{2}+c_{1} d+e_{1} & \text { when } d \in\left[0, d_{c}[\right. \\ a_{2} d^{3}+b_{2} d^{2}+c_{2} d+e_{2} & \text { when } d \in\left[d_{c}, 1\right]\end{cases}
$$

where $\left.d_{c} \in\right] 0,1\left[\right.$ and with the following continuity conditions at $d \in\left\{0, d_{c}, 1\right\}$ :

$$
\begin{aligned}
& \nu_{-}(0)=\nu_{0} \text { and } \nu_{-}^{\prime}(0)=0 \\
& \nu_{-}\left(d_{c}^{-}\right)=\nu_{-}\left(d_{c}^{+}\right)=\nu_{c} \text { and } \nu_{-}^{\prime}\left(d_{c}^{-}\right)=\nu_{-}^{\prime}\left(d_{c}^{+}\right)=\nu_{c}^{\prime} \\
& \nu_{-}\left(d_{f}\right)=1 / 2 \text { and } \nu_{-}^{\prime}\left(d_{f}\right)=\nu_{f}^{\prime}
\end{aligned}
$$

Note that there are eight unknowns $\left(a_{i}, b_{i}, c_{i}, e_{i}\right)_{i=1,2}$ and eight necessary continuity conditions for the corresponding problem to be well-posed. Here, we adjust the values $d_{c}$ to the minimum of the bulk modulus $K_{-}$from the experimental data $\left(d_{i}, K_{i}\right)_{1 \leqslant i \leqslant n}$ while $\nu_{c}$ is adjusted from the Poisson's ratio $\left(d_{i}, \nu_{i}\right)_{1 \leqslant i \leqslant n}$. The derivative $\nu_{c}^{\prime}$ is given by the condition $K_{-}^{\prime}\left(d_{c}\right)=0$ i.e. from a change of sign in $(19 \mathrm{c}): \nu_{c}^{\prime}=\left(1-2 \nu_{c}\right) E_{0} /\left(2 E\left(d_{c}\right)\right)$. Finally, we adjust $\nu_{f}^{\prime}$ based on the Poisson's ratio data for the slope of $\nu_{-}$at $d=d_{f}$. Results are shown on Fig. 3 and the adjustment parameters are provided in Table. 1. Using this fit, we are able to compute $\nu_{-}$and its derivative for any $d \in\left[0, d_{f}\right]$ and then to evaluate the radii $a_{-}(d)$ and $b_{-}(d)$ from (21a)-(21b): Fig. 2.middle-left presents the envelope of the damage criterion for various damage values while Figs. 2.bottom plot the radii. Ellipses are obtained in the traction half-plane, which shrink when damage increases. In the compression half-plane, the obtained envelope is an ellipse for $d \in\left[0, d_{c}\left[\right.\right.$. At the transition $d=d_{c}$, the bulk modulus $K_{-}$ is minimal and its derivative vanishes while the damage criterion (18) degenerates to

$$
|\operatorname{dev}(\boldsymbol{\sigma})| \geqslant a(d)
$$

This means that the damage criterion reduces to a von Mises criterion on the deviatoric part of the stress tensor. The shape of the envelope changes to an hyperbola for $\left.d \in] d_{c}, d_{f}\right]$. This corresponds to the case 2 on Fig. 1 .

Fig. 4 shows similar results for a Darley Dale sandstone. As for the previous Etna basalt, there is a transition of the damage envelope from an ellipse to an hyperbola in the compression stress half-space.

Finally, we consider the Icelandic basalt presented on Fig. 5.top-left by its experimental measurements of the Young modulus and Poisson's ratio during a similar experiment, where a cylindrical sample undergoes cycles of compression. Figs. 5.top-right and 5.middle-right plots the bulk modulus $K_{-}$and the Poisson's ratio $\nu_{-}$versus $d$. Poisson's ratio is approximately a constant function of $d$. Thus, by its definition (14c), the bulk modulus $K_{-}$is linear and also convex. From theorem 5 the Helmholtz free energy is also convex with respect to the two state 
variables separately. Next, from theorem 7, the damage criterion in the stress space is always an ellipse. This corresponds to the case 1 on Fig. 1. Fig. 5.middleleft plots the damage criterion in the stress space, while Figs. 5.bottom shows the radii.

\section{Bulk modulus derived from theory in traction}

In traction, as pointed out in the previous paragraph, experimental observations show that the damage envelopes present an ellipsoidal shape (see e.g. Lemaitre and Chaboche 1990, p. 183). Choosing a constant Poisson's ratio $\nu_{+}(d)=\nu_{0}$ is consistent with these observation: thanks to remark 8 , we recover an ellipsoidal shape of the damage criterion. Nevertheless, more involved choices are possible in traction. Ponte-Castañeda and Willis (1995), eqn (4.16), proposed explicit expressions for the shear $G(d)$ and bulk modulus $K(d)$ versus damage $d$ from a micro-mechanical analysis, considering a distribution of randomly oriented pennyshaped micro-cracks with a spherical spatial distribution (see also Kondo et al. 2007, eqns (32)-(33)):

$$
\begin{aligned}
& G_{+}(d)=G_{0}\left(1-\frac{120\left(1-\nu_{0}\right)\left(5-\nu_{0}\right) d}{225 \pi\left(2-\nu_{0}\right)+16\left(4-5 \nu_{0}\right)\left(5-\nu_{0}\right) d}\right) \\
& K_{+}(d)=K_{0}\left(1-\frac{12\left(1-\nu_{0}^{2}\right) d}{9 \pi\left(1-2 \nu_{0}\right)+4\left(1+\nu_{0}\right)^{2} d}\right)
\end{aligned}
$$

where $G_{0}=E_{0} /\left(2\left(1+\nu_{0}\right)\right)$ and $K_{0}=E_{0} /\left(3\left(1-2 \nu_{0}\right)\right)$ are respectively the shear and bulk modulus of the undamaged material. An investigation of expression (23b) shows that $K_{+}$is strictly convex versus $d$ for any $\left.\nu_{0} \in\right] 0,1 / 2[$ (see also Fig. 6.topright). Recall that the Young modulus and Poisson's ratio write respectively from bulk and shear modulus as $E=9 K G /(3 K+G)$ and $\nu=(3 K-2 G) /(6 K+2 G))$, see e.g. Slaughter (2002), p. 215. Another investigation of the obtained expression for the Young modulus $E$ shows that it is always positive and also strictly convex versus $d$, for any $\nu_{0} \in[0,1 / 2$ [ (see also Fig. 6.top-left). Note that $E$ is here nonaffine: thus the definition of the damage variable $d$ do not coincides Kachanov's definition (20). From the convexity of both $E$ and $K_{+}$, from theorem 5 , in traction, the Helmholtz free energy is convex with respect to the two state variables separately. Fig. 6.middle-right plots Poisson's ratio: observe that it is decreasing, as expected in traction. Next, from (19a)-(19b), we deduce expressions of the radii $a_{+}$and $b_{+}$in traction. An exploration of these expressions shows that both $a_{+} \geqslant 0$ and $b_{+} \geqslant 0$ for any $d \in[0,1]$ and $\left.\nu_{0} \in\right] 0,1 / 2[$ (see also Fig. 6.bottom). Consequently, the shape of the damage criterion is an ellipse, as shown on Fig. 6.middle-left. Note that this model prediction is consistent with experimental observations (see e.g. Lemaitre and Chaboche 1990, p. 183). 

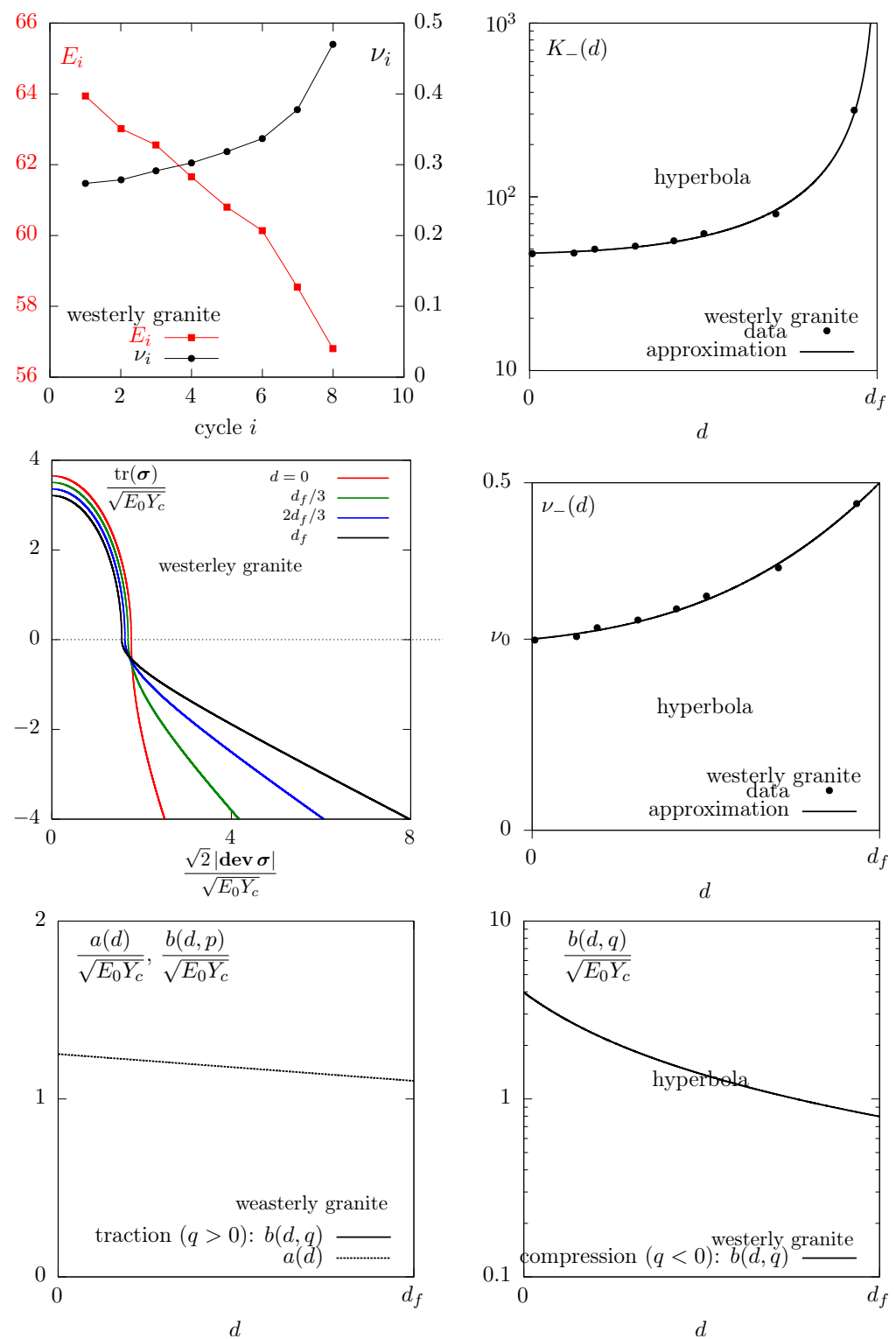

Figure 2. Westerly granite (experimental data from Heap et al. (2010), Fig. 3f). (top-left) experimental measurement of the Young modulus $E_{i}$ and the Poisson's ratio $\nu_{i}$ versus compression cycle $i$; (top-right) Bulk modulus $3 K=(1-2 \nu) / E$ vs $d$; (middle-left) Envelope of the damage criterion vs $d$; (middle-right) Poisson's ratio versus the damage variable $\left(\nu_{i}, d_{i}\right)$ : experimental data and its spline approximation $\nu(d)$; (bottom) Radius $a(d)$ and $b(d, q)$ vs $d$ in traction $(q>0)$ and compression $(q<0)$. 

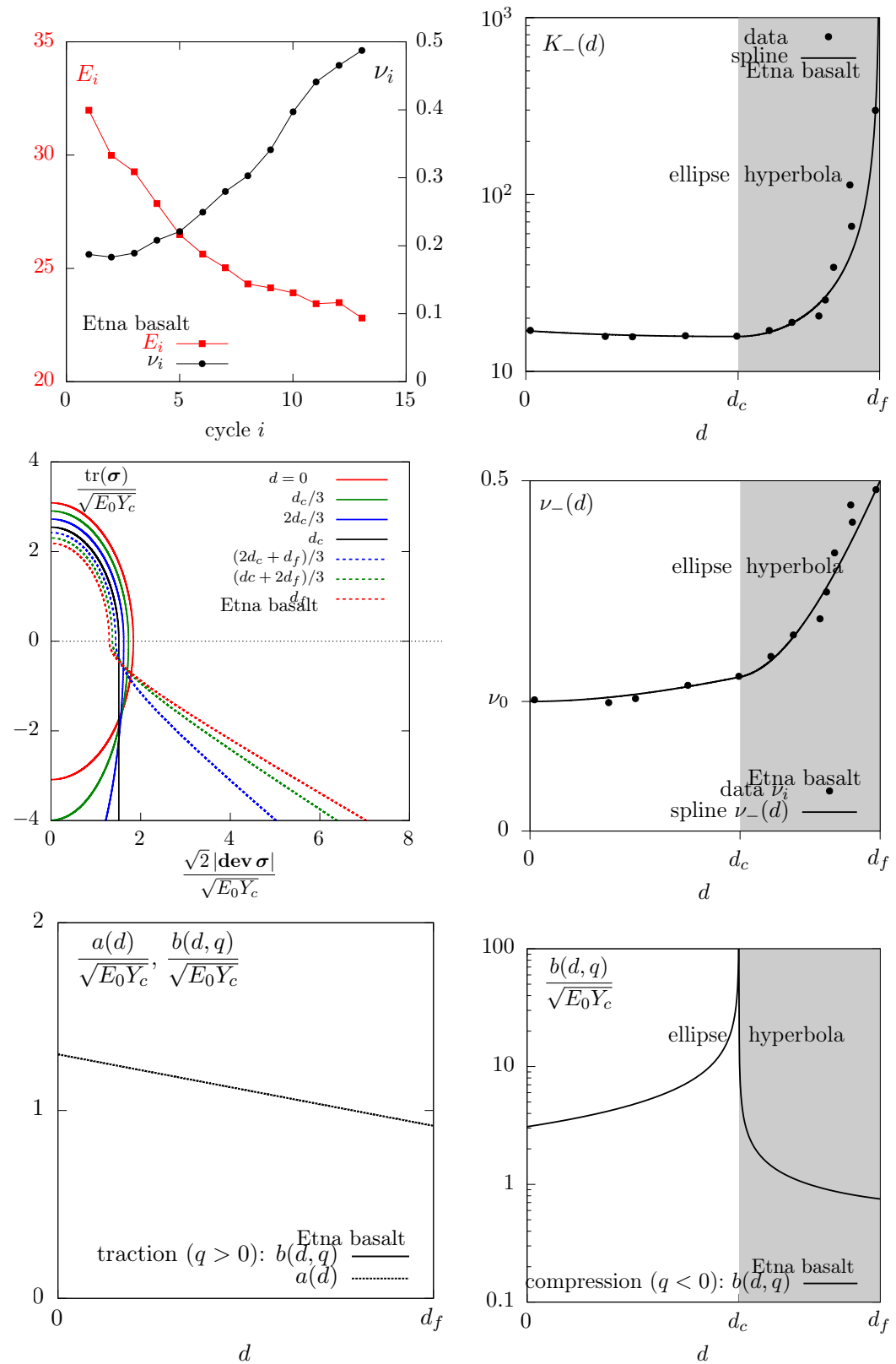

Figure 3. Similar analysis for the Etna basalt (experimental data from Heap et al. (2009), Fig. 7a). 

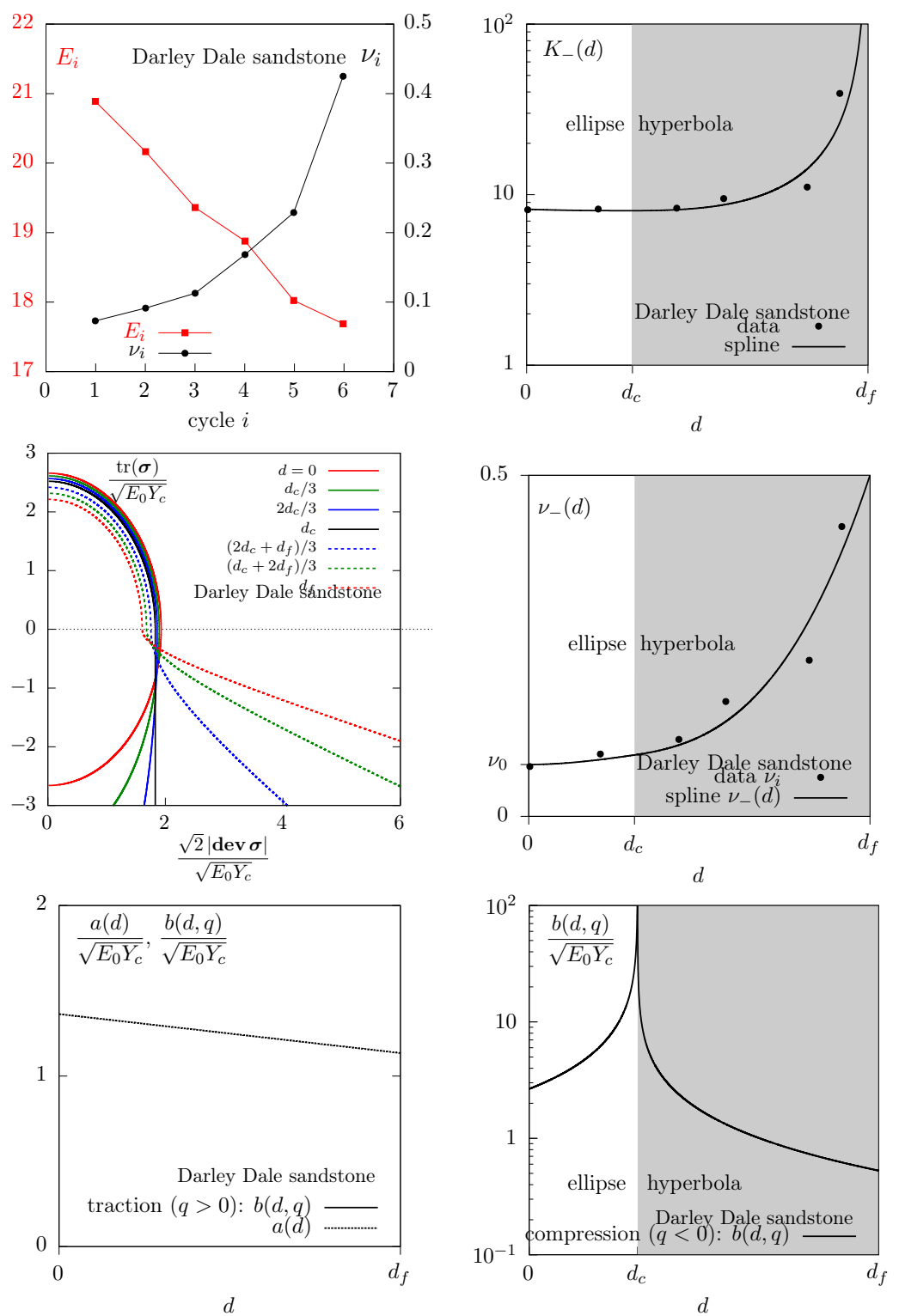

Figure 4. Similar analysis for the Darley Dale sandstone (experimental data from Heap et al. (2010), Fig. 3e). 

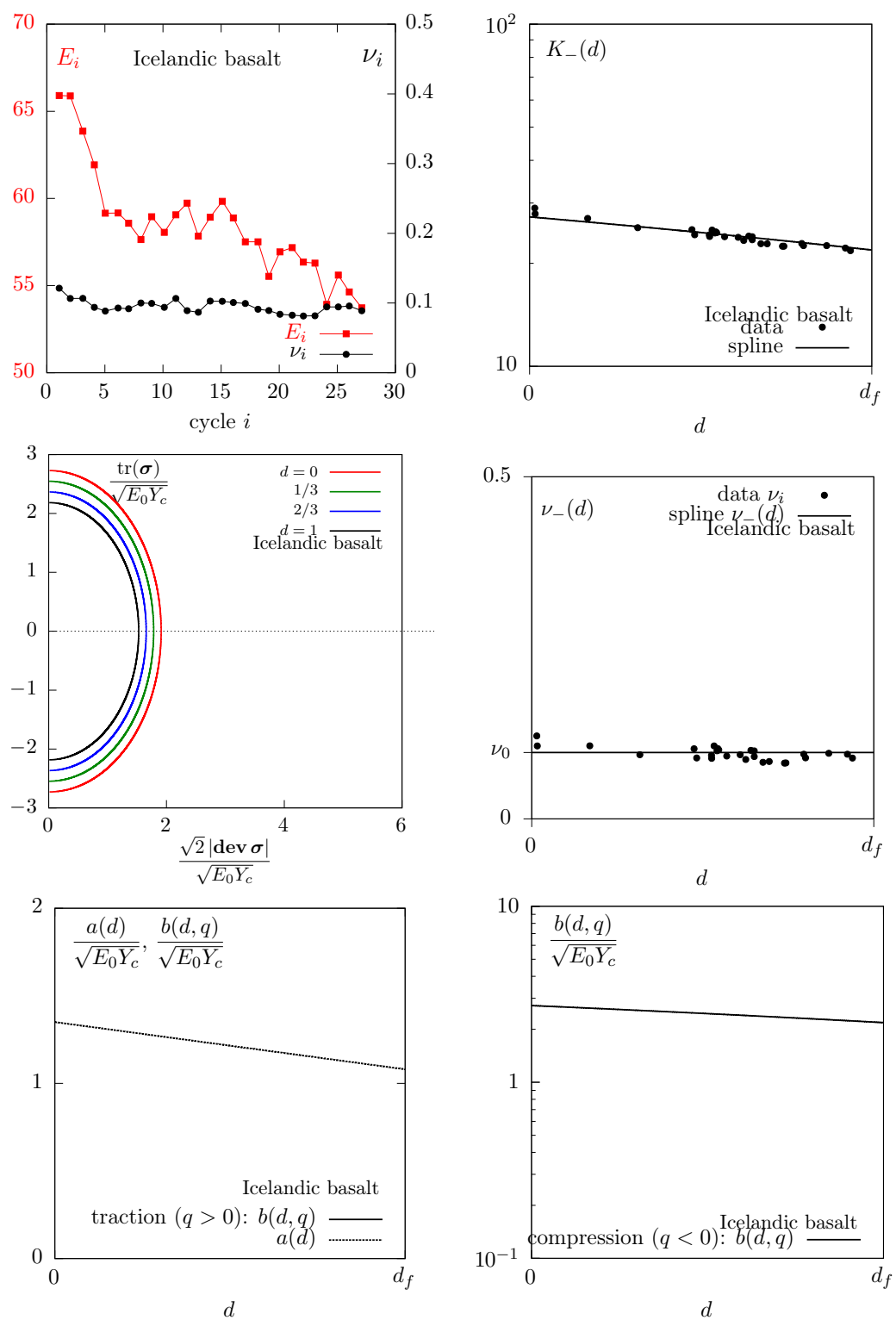

Figure 5. Similar analysis for the Icelandic basalt (experimental data from Heap et al. (2010), Fig. 3c). 

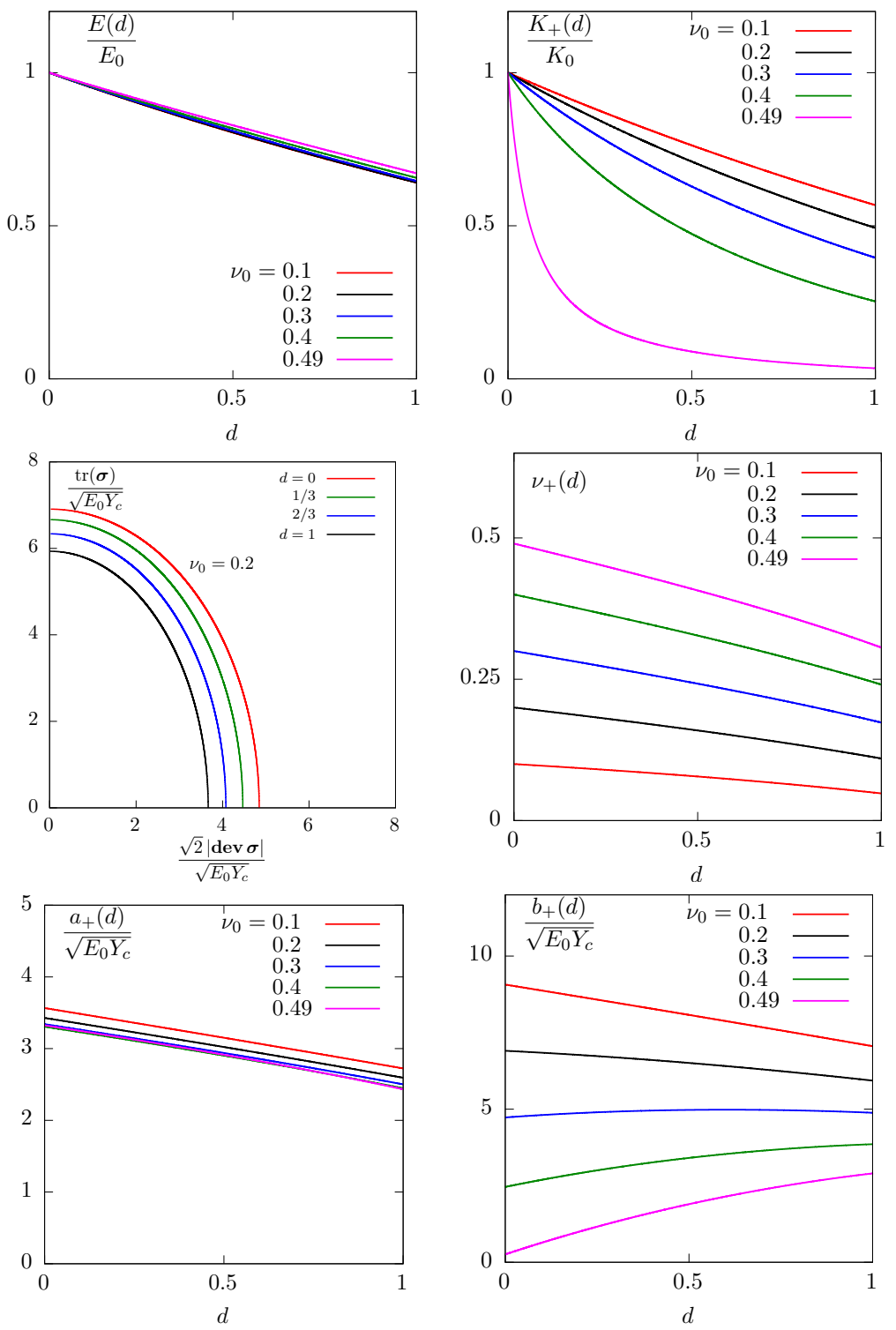

Figure 6. Traction: expressions derived from micro-crack theory. (top) shear $E$ and bulk modulus $K_{+}$versus $d$; (middle-left) Envelopes of the damage criterion vs $d$ for $\nu_{0}=0.2$; (middle-right) Poisson's ratio versus $d$; (bottom) Radius $a_{+}(d)$ and $b_{+}(d)$ vs $d$. 


\section{Interpretation and discussion}

To interpret the results of Figs. 2 to 5, rocks properties, and particularly their initial porosity, are worth considering. At first glance, the increase in bulk modulus, $K$, with the damage, $d$, observed after an initial stage in which $K$ is almost constant or weakly decreasing in most of the rocks analyzed here (case 2 on Fig. 1), (with the exception of the Icelandic basalt, Fig. 5), appears surprising.

A possible interpretation for this behavior is a damage-induced collapse of pores in rocks under compression. In rocks with a significant initial porosity, such as sandstones or Etna basalt, the closing of pores during compression is expected to lead to local contracting strains, to a decrease in porosity, i.e. to compaction (Fortin et al. 2009), and to an increase of $P$ and $S$ seismic wave velocities (Fortin et al. 2011). Recent X-ray micro-tomography observations of damage and deformation of rocks during multiaxial compression evidenced these mechanisms in various porous rocks (Renard et al. 2017), including Etna basalt (McBeck et al. 2019). In these materials, when the confining pressure is large, this compaction phase can last almost until the so-called cataclastic compaction failure (Fortin et al. 2009) phase. Compaction is however much reduced under small (or zero) confinement conditions, for which failure occurs through shear localization and faulting accompanied by dilatancy. Such a compaction phase induced by pore collapse could explain the increase of $K$ above $d_{c}$.

In materials following case 2 on Fig. 1, this would indicate that in the initial stage of damage $\left(d<d_{c}\right)$, another damage mechanism inducing a decrease of $K$, such as micro-cracking, competes with the pore collapse mechanism. Note that this initial stage is accompanied in these materials by a sharp decrease of the Young's modulus, E, with the number of loading cycles (see e.g. Fig. 3.a). while the evolution above $d_{c}$ is much slower. During the increase of $K$, the damage envelope in the compression half-space expands, meaning that further damaging becomes more and more unlikely as damage accumulates. This could be interpreted as a progressive closure of the pores, the weakest closing first, under relatively smaller stresses, and the strongest requiring much larger stresses.

This scenario would imply that damage events (pore collapses) are not significantly interacting mechanically and therefore are randomly distributed (as opposed to correlated) within the rock. It is supported by X-ray tomography observations, at least in the pre-faulting phase (McBeck et al. 2019). In this context, the particular behavior of Icelandic basalt (case 1 on Fig. 1 ; and Fig. 5), for which this $K$-increasing phase is not observed, could be related to its low porosity $(1.2 \%)$ and extremely homogeneous crystalline structure that does not present visible pre-existing micro-cracks (Heap et al. 2010).

Several questions remains open, however. First, the proposed interpretation hardly explains the behavior of Westerly granite, a low-porosity material of about $0.8 \%$ 
that nevertheless belongs to case 3 on Fig. 1. In addition, the compression experiments of Heap et al. $(2009,2010)$ that we used to build Figs. 2 to 5 were performed under uni-axial loading, without confinement. In this case, one would expect a shear faulting failure, with a limited compaction and pore collapse phase before the onset of dilatancy due to localized micro-cracking (Fortin et al. 2009). Such dilatant phase should be accompanied by a decreasing bulk modulus $K$ in the latest stages of damage and deformation, which is not recovered in our analysis. Overall, an increasing bulk modulus as approaching compressive failure, such as case 2 and 3 of Fig. 1 above, appear unphysical.

To explain these shortcomings, we note that our approach assumes (i) a spatially homogeneous and (ii) isotropic damage. Isotropy is a rough assumption for compressive failure under low confinement. Indeed, at least in the first stages of deformation and damage, microcracks tends to align along the maximum principal compressive stress (Renard et al. 2018). Such anisotropic damage might explain a large increase of $\nu$ when measured perpendicularly to this axis (Heap et al. 2010). In addition, except for cataclastic compaction failure under high confinement, compressive failure is generally preceded by a progressive localization of damage and deformation along an inclined fault (Lockner et al. 1991; Renard et al. 2017), including for contracting strains in porous rocks (McBeck et al. 2019). Such localization is not taken into account in our approach, which therefore likely fails to describe these final stages of compressive failure.

\section{Comparison of the damage criterion with stress measurements}

In this section, we compare our derived damage yield criterion, $Y<Y_{c}$, as expressed in the Cauchy stress space to available measurements of stresses in another geomaterial, sea ice, and discuss its agreement with the previously deducted damage criteria.

Over certain time and space scales, ice behaves in the quasi-brittle regime in a manner very similar to rocks (Schulson et al. 2006b; Weiss et al. 2007; Marsan and Weiss 2010). Both fresh ice at the laboratory scale and sea ice at the scale of the Arctic ocean have therefore been modeled using a combination of damage mechanics and stress yield criterion (Girard et al. 2010, 2011).

In the last decades, measurements of in-situ stresses within the sea ice cover have been made to study its mechanical behavior (Richter-Menge et al. 2002). While reconstructing failure envelopes in the laboratory require making repetitive estimates of principal stresses at failure under different compressive ratios over many samples (Haied et al. 2000; Schulson 2001; Schulson et al. 2006a,b; Haimson and Rudnicki 2010), such in situ measurements allow deducting the failure envelope of ice over the entire Cauchy stress space.

Here, in situ stress measurements made using biaxial stressmeters specifically designed for sea ice applications (Johnson and Cox 1982) are presented. Owing to 

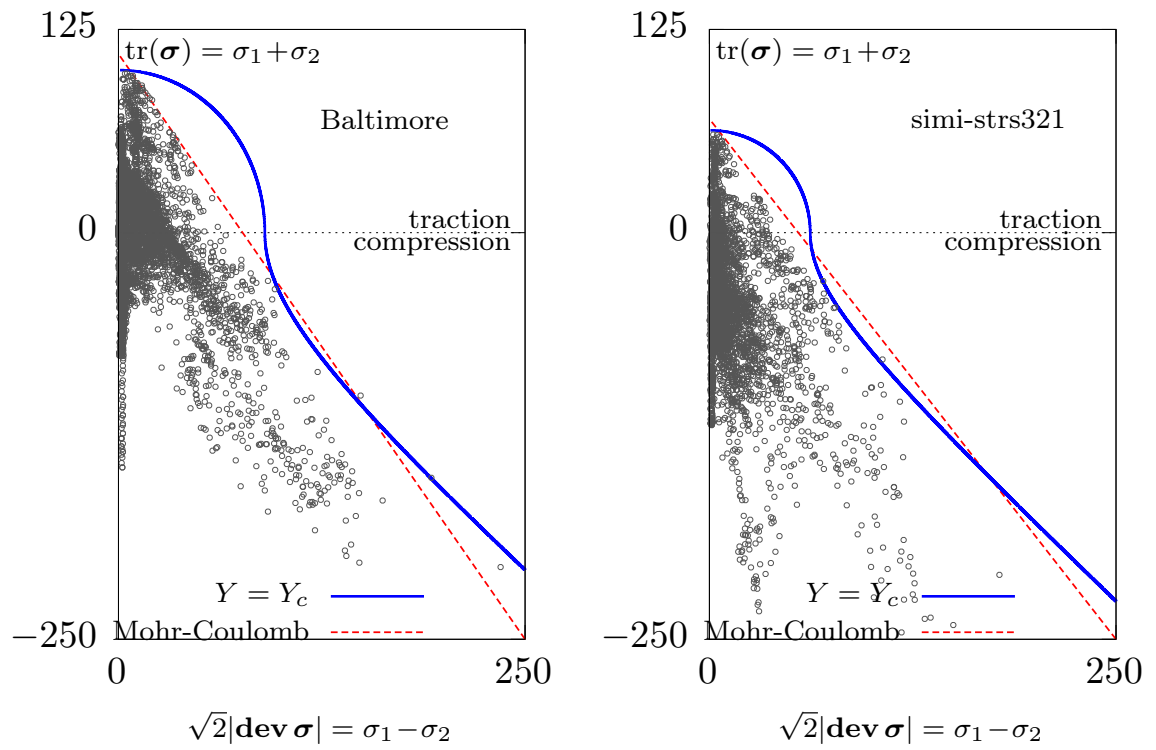

Figure 7. The $Y=Y_{c}$ damage criterion graphically expressed in terms of the Cauchy stress tensor $\boldsymbol{\sigma}$ (continuous blue line). Comparison with experimental data from Weiss et al. (2007), Fig. 2.a where units are in MPa. The radii of the ellipse and the hyperbola are adjusted to the data: (left) Baltimore: $\sqrt{2} a=90 \mathrm{Mpa}, b_{+}=100 \mathrm{Mpa}, b_{-}=80 \mathrm{MPa}$; (right) simi-strs321: $\sqrt{2} a=31 \mathrm{Mpa}, b_{+}=63 \mathrm{Mpa}, b_{-}=58 \mathrm{MPa}$. The Mohr-Coulomb damage criterion used in Weiss et al. (2007) is also indicated by the dotted red line.

the huge aspect ratio between lateral extension and thickness of the sea ice cover, plane stress conditions are fulfilled, therefore local in-plane stresses are recorded. The data reported on Fig. 7 is from two stressmeters deployed on the Beaufort Sea from October 1997 to July 1998 (Richter-Menge et al. 2002). Measurements performed on other sensors gave similar results. Each data point represents a oneminute averaged measure, with a sampling frequency of one hour. Overall, about 6500 stress states are represented on each plot. As the sea ice cover experienced a large variety of external forcings (essentially due to the winds) both in terms of intensity and direction over the 1 year period covered by the measurements, we can reasonably assume that the data fills the local failure envelope of sea ice.

Fig. 7 represents these in situ measurements together with the envelope of the derived damage criterion, $Y<Y_{c}$, in the space of the two eigenvalues, $\sigma_{1}>\sigma_{2}$ of the Cauchy stress tensor in the bidimensional geometry of the sea ice cover. Here $b_{ \pm}$denotes the second radius of the ellipse and of the hyperbola. The radii of the envelope are adjusted to the data. 
Fig. 7 also compares the envelope with the Mohr-Coulomb criterion, which in the view of this stress data was interpreted by (Weiss et al. 2007; Weiss and Schulson 2009) as a suitable damage criteria for sea ice.

However here, we observe that both damage criteria are in good agreement with the measurements. As the shape of both envelopes are analogous, one can argue that they would lead to similar results in numerical simulations of progressive damage. However, the physical concepts behind both criteria differ dramatically.

While the Mohr-Coulomb (Coulomb 1773; Mohr 1900) criterion is based on the ill-defined concept of internal friction (Savage et al. 1996), the criterion derived here is thermodynamically-based and uses the strain energy release rate $Y$. By this fact, this new criterion presents several advantages.

Unlike the Mohr-Coulomb criterion, it indeed links to the evolution of the Poisson's ratio with damage observed in laboratory experiments. Unlike the Mohr-Coulomb criterion also, the theory of which is restricted to compressive stresses, it includes an unilateral extension that distinguishes between traction and compression. It also allows for friction and plasticity to be introduced in (1): this would generates additional dissipation terms but the damage mechanism would remains linked to the strain energy release rate. The present comparison with in situ stress measurements therefore suggests that the damage criterion derived here can constitute a valuable alternative to the Mohr-Coulomb criterion in geophysical applications.

\section{Conclusion}

In this work, we have derived a new damage criterion that (1) verifies the second principle of thermodynamics, (2) distinguishes between compressive and tensile states of stress and (3) accounts for the evolution of the Poisson's ratio with the level of damage of the material. We have expressed this criterion in the stress space and compared it with in-situ stress measurements. Under compression, the good agreement found between the criterion, the data and the Mohr-Coulomb criterion compels to a reinterpretation of the concept of damage criterion. Comparisons of the derived evolution of the Poisson's ratio and bulk modulus as a function of the damage with laboratory experiments on rocks has however shown that the criterion fails to capture some of the aspects of the evolution of the bulk modulus, in particular, the observed decrease in the dilatant phase that characterizes the final stages of compressive failure. These shortcomings are likely the consequence of the isotropic damage assumption made here and call for a future extension of this work to anisotropic damage. Nevertheless, we argue that the criterion derived here presents several advantages over the widely used Mohr-Coulomb criterion and is more physically sound is several aspects. Future work will consider numerical simulations of progressive damage, in order to assess the present model in traction and compression. 


\section{References}

Abe S (2016) Comparison of discrete element simulations to theoretical predictions of the elastic moduli of damaged rocks. Int. J. Rock Mech. Min. Sci. 88: 265-272.

Amitrano D (2003) Brittle-ductile transition and associated seismicity: experimental and numerical studies and relationship with the $b$ value. J. Geophys. Res. Solid Earth 108(B1): 2044.

Amitrano D, Grasso JR and Hantz D (1999) From diffuse to localised damage through elastic interaction. Geophys. Res. Lett. 26: 2109-2112.

Ashby MF and Sammis CG (1990) The damage mechanics of brittle solids in compression. Pure Appl. Geophys. 133(3): 489-521.

Berthier E, Démery V and Ponson L (2017) Damage spreading in quasi-brittle disordered solids: I. localization and failure. J. Mech. Phys. Solids 102: 101-124.

Besson J, Cailletaud G, Chaboche JL, Forest S and Blétry M (2010) Non-linear mechanics of materials. Springer.

Clarke FH (1990) Optimization and nonsmooth analysis. Philadelphia, USA: SIAM.

Coulomb CA (1773) Essai sur une application des règles de maximis et minimis à quelques problèmes de statique relatifs à l'architecture. Acad. Roy. Sci. Mem. Math. Phys. 7: 343-387.

Erdogan F and Sih GC (1963) On the crack extension in plates under plane loading and transverse shear. J. Basic Eng. 85(4): 519-525.

Eslami J, Grgic D and Hoxha D (2010) Estimation of the damage of a porous limestone from continuous (P-and S-) wave velocity measurements under uniaxial loading and different hydrous conditions. Geophys. J. Int. 183(3): 1362-1375.

Fortin J, Stanchits S, Dresen G and Gueguen Y (2009) Acoustic emissions monitoring during inelastic deformation of porous sandstone: comparison of three modes of deformation. Pure Appl. Geophys. 166(5-7): 823-841.

Fortin J, Stanchits S, Vinciguerra S and Gueguen Y (2011) Influence of thermal and mechanical cracks on permeability and elastic wave velocities in a basalt from Mt. Etna volcano subjected to elevated pressure. Tectonophysics 503(1-2): 60-74.

Girard L, Amitrano D and Weiss J (2010) Failure as a critical phenomenon in a progressive damage model. J. Stat. Mech. Theory Exp. 2010(01): P01013.

Girard L, Bouillon S, Weiss J, Amitrano D, Fichefet T and Legat V (2011) A new modeling framework for sea-ice mechanics based on elasto-brittle rheology. Annals Glaciology 52(57): 123-132.

Griffith AA (1921) The phenomena of rupture and flow in solids. Phil. Trans. R. Soc. Lond. A 221(582-593): 163-198.

Grindrod PM, Heap MJ, Fortes AD, Meredith PG, Wood IG, Trippetta F and Sammonds PR (2010) Experimental investigation of the mechanical properties of synthetic magnesium sulfate hydrates: implications for the strength of hydrated deposits on Mars. J. Geophys. Res. Planets 115(E6).

Haied A, Kondo D and Henry JP (2000) Strain localization in Fontainebleau sandstone. Mech. Cohes.-Frict. Mater. 5(3): 239-253. 
Haimson B and Rudnicki JW (2010) The effect of the intermediate principal stress on fault formation and fault angle in siltstone. J. Struct. Geol. 32(11): 1701-1711.

Halphen B and NGuyen QS (1975) Sur les matériaux standards généralisés. J. Méca. 14: $39-63$.

Hayakawa K and Murakami S (1997) Thermodynamical modeling of elastic-plastic damage and experimental validation of damage potential. Int. J. Damage Mech. 6(4): 333-363.

Heap MJ, Faulkner DR, Meredith PG and Vinciguerra S (2010) Elastic moduli evolution and accompanying stress changes with increasing crack damage: implications for stress changes around fault zones and volcanoes during deformation. Geophys. J. Int. 183(1): 225-236.

Heap MJ, Vinciguerra S and Meredith PG (2009) The evolution of elastic moduli with increasing crack damage during cyclic stressing of a basalt from Mt. Etna volcano. Tectonophysics 471(1-2): 153-160.

Jaeger JC, Cook NGW and Zimmerman R (2007) Fundamentals of rock mechanics. fourth edition. Blackwell.

Johnson JB and Cox GFN (1982) Stress sensor particularly suitable for elastic, plastic and visco-elastic materials. United States patent \#4346600.

Kachanov LM (1958) Time of the rupture process under creep conditions. Izv. Akad. Nauk SSSR, Otd. Tekhn. Nauk 8: 26-31.

Kondo D, Welemane H and Cormery F (2007) Basic concepts and models in continuum damage mechanics. Revue européenne de génie civil 11(7-8): 927-943.

Ladevèze P and Lemaitre $\mathrm{J}$ (1984) Damage effective stress in quasi-unilateral conditions. In: Theoretical and applied mechanics. XVIth international congress of theoretical and applied mechanics, Lyngby, Denmark. Elsevier.

Lemaitre J (1985) A continuous damage mechanics model for ductile fracture. J. Eng. Mat. Tech. 107(1): 83-89.

Lemaitre J (1996) A course on damage mechanics. Second edition. Springer.

Lemaitre J and Chaboche JL (1990) Mechanics of solid materials. UK: Cambridge university press.

Lockner DA, Byerlee JD, Kuksenko V, Ponomarev A and Sidorin A (1991) Quasi-static fault growth and shear fracture energy in granite. Nature 350(6313): 39.

Marsan D and Weiss J (2010) Space/time coupling in brittle deformation at geophysical scales. Earth Planet. Sci. Lett. 296(3-4): 353-359.

McBeck JA, Cordonnier B, Vinciguerra S and Renard F (2019) Volumetric and shear strain localization in Mt. Etna basalt. Geophys. Res. Lett. 46.

Mohr CO (1900) Welche umstände bedingen die elastizitätsgrenze und den bruch eines materials ? Zeitschrift des Vereines deutscher Ingenieure 44: 1524-1530, 1572-1577.

Murakami S (2012) Continuum damage mechanics: a continuum mechanics approach to the analysis of damage and fracture. Springer.

Ponte-Castañeda P and Willis JR (1995) The effect of spatial distribution on the effective behavior of composite materials and cracked media. J. Mech. Phys. Solids 43(12): 
1919-1951.

Renard F, Cordonnier B, Kobchenko M, Kandula N, Weiss J and Zhu W (2017) Microscale characterization of rupture nucleation unravels precursors to faulting in rocks. Earth Planet. Sci. Lett. 476: 69-78.

Renard F, Weiss J, Mathiesen J, Ben-Zion Y, Kandula N and Cordonnier B (2018) Critical evolution of damage toward system-size failure in crystalline rock. $J$. Geophys. Res. Solid Earth 123(2): 1969-1986.

Richter-Menge JA, McNutt SL, Overland JE and Kwok R (2002) Relating arctic pack ice stress and deformation under winter conditions. J. Geophys. Res. Oceans 107(C10).

Riva F, Agliardi F, Amitrano D and Crosta GB (2018) Damage-based time-dependent modeling of paraglacial to postglacial progressive failure of large rock slopes. $J$. Geophys. Res. Earth Surf. 123(1): 124-141.

Rockafellar RT (1974) Conjugate duality and optimization. Philadelphia, USA: SIAM.

Sammis CG and Ashby MF (1986) The failure of brittle porous solids under compressive stress states. Acta Metall. 34(3): 511-526.

Saramito P (2016) Complex fluids: modelling and algorithms. Springer.

Savage JC, Byerlee JD and Lockner DA (1996) Is internal friction friction? Geophys. Res. Lett. 23(5): 487-490.

Schulson EM (2001) Brittle failure of ice. Eng. Fracture Mech. 68(17-18): 1839-1887.

Schulson EM, Fortt AL, Iliescu D and Renshaw CE (2006a) Failure envelope of first-year Arctic sea ice: The role of friction in compressive fracture. J. Geophys. Res. Oceans 111(C11S25).

Schulson EM, Fortt AL, Iliescu D and Renshaw CE (2006b) On the role of frictional sliding in the compressive fracture of ice and granite: terminal vs. post-terminal failure. Acta Materialia 54(15): 3923-3932.

Slaughter WS (2002) The linearized theory of elasticity. Springer.

Weiss J and Schulson EM (2009) Coulombic faulting from the grain scale to the geophysical scale: lessons from ice. J. Phys. D 42(21): 214017.

Weiss J, Schulson EM and Stern HL (2007) Sea ice rheology from in-situ, satellite and laboratory observations: fracture and friction. Earth Planet. Sci. Lett. 255(1-2): 1-8.

Yang SQ, Ranjith PG, Huang YH, Yin PF, Jing HW, Gui YL and Yu QL (2015) Experimental investigation on mechanical damage characteristics of sandstone under triaxial cyclic loading. Geophys. J. Int. 201(2): 662-682. 